\title{
Reversible Protein Phosphorylation Regulates Circadian Rhythms
}

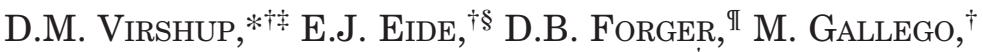 \\ AND E. VIELHABER HARNISH ${ }^{\dagger * *}$ \\ *Division of Hematology/Oncology, Department of Pediatrics, University of Utah, Salt Lake City, Utah 84112; \\ Department of Oncological Sciences and the Huntsman Cancer Institute, University of Utah, Salt Lake City, \\ Utah 84112; "Department of Mathematics, University of Michigan, Ann Arbor, Michigan 48109
}

\begin{abstract}
Protein phosphorylation regulates the period of the circadian clock within mammalian cells. Circadian rhythms are an approximately 24-hour cycle that regulates key biological processes. Daily fluctuations of wakefulness, stress hormones, lipid metabolism, immune function, and the cell division cycle are controlled by the molecular clocks that function throughout our bodies. Mutations in regulatory components of the clock can shorten or lengthen the timing of the rhythms and have significant physiological consequences. The clock is formed by a negative feedback loop of transcription, translation, and inhibition of transcription. The precision of clock timing is controlled by protein kinases and phosphatases. Casein kinase I $\varepsilon$ is a protein kinase that regulates the circadian clock by periodic phosphorylation of the proteins PER1 and PER2, controlling their stability and localization. The role of phosphorylation in regulating PER function in the clock has been explored in detail. Quantitative modeling has proven to be very useful in making important predictions about how changes in phosphorylation alter the clock's behavior. Quantitative data from biological studies can be used to refine the quantitative model and make additional testable predictions. A detailed understanding of how reversible protein phosphorylation regulates circadian rhythms and a detailed quantitative model that makes clear, testable, and accurate predictions about the clock and how we may manipulate it can have important benefits for human health. Pharmacological manipulation of rhythms could mitigate stress from jet lag, shift work, and perhaps even seasonal affective disorder.
\end{abstract}

\section{INTRODUCTION}

Circadian rhythms govern key physiologic processes, ranging from sleep-wake cycles, glucose, lipid and drug metabolism, heart rate, stress and growth hormones, and immunity, as well as basic cellular processes such as DNA repair and the timing of the cell division cycle (Chang and Reppert 2001; Fu et al. 2002; Reppert and Weaver 2002; Matsuo et al. 2003; Lowrey and Takahashi 2004; Antoch et al. 2005; Turek et al. 2005). The disruption of circadian rhythms causes significant physiologic stress, is frequently experienced in jet lag and night shift work, and has been linked to bipolar disorder (Mansour et al. 2005). Circadian changes in physiology can also be exploited to more efficiently treat disease. For example, because there is a fourfold increase in cholesterol biosynthesis in the liver at night, the cholesterol-lowering 3hydroxy-3-methylglutaryl-coenzyme A (HMG-CoA) reductase inhibitors (statins such as lovastatin) are most effective when taken in the evening (e.g., see Wallace et al. 2003). Similarly, two studies found that children with leukemia who take oral maintenance chemotherapy (6mercaptopurine and methotrexate) in the evening have a clear survival advantage over children who take these medications in the morning (Rivard et al. 1993; Schmiegelow et al. 1997). Mice with mutant circadian

Present addresses: ${ }^{\star}$ Program in Cancer and Stem Cell Biology, DukeNUS Graduate Medical School Singapore, 2 Jalan Bukit Merah, Singapore 169547; ${ }^{\S}$ Fred Hutchison Cancer Research Center, Seattle, Washington 98109; **Sanofi-Aventis, Bridgewater, New Jersey 08807. rhythms are at increased risk of obesity, metabolic syndrome, and cancer (Fu et al. 2002; Antoch et al. 2005; Turek et al. 2005). Thus, circadian regulation of physiology has many important consequences for health. A detailed quantitative model that makes clear, testable, and accurate predictions about the clock and how we may manipulate it can have important benefits for human health. Model predictions can guide drug development. Pharmacological manipulation of rhythms could mitigate stress from jet lag, shift work, and perhaps even seasonal affective disorder. The best drug targets in the clock are enzymes, and the enzymes that have the greatest effect on the clock are kinases, specifically casein kinase I (CKI).

Reversible protein phosphorylation is a key regulator of circadian rhythms. Mutations in PERIOD, TIMELESS, $C L O C K, C Y C L E$, and the casein kinase IE (CKIE) ortholog DOUBLETIME all alter circadian rhythm in Drosophila. Orthologs of the same genes regulate mammalian circadian rhythms. Similarly, the first mammalian circadian rhythms mutant identified, the tau hamster, also has a missense mutation in CKIع (Ralph and Menaker 1988; Lowrey et al. 2000). This tau mutation, R178C, decreases the $V_{\max }$ of CKIE eightfold. Mutations both in the closely related and apparently redundant CKI $\delta$ and in a CKI phosphorylation site in PER2, have been identified in human families with familial advanced sleep phase syndrome (FASPS) (Jones et al. 1999; Toh et al. 2001; Xu et al. 2005). However, we are only beginning to understand how these kinase mutations alter circadian period. One confusing point has been that mutations in Drosophila CKI (Doubletime, Dbt) that either cause long 
or short periods all decrease CKI activity in vitro (Suri et al. 2000; Eide and Virshup 2001; Preuss et al. 2004). Likewise, mutants with decreased mammalian CKIE and $\mathrm{CKI} \delta$ activity (in vitro) may either lengthen (Eide et al. 2005) or shorten circadian periods (Lowrey et al. 2000; $\mathrm{Xu}$ et al. 2005). It has been difficult to reconcile how different mutations and inhibitors that apparently decrease CKI activity have opposite effects on circadian periods by previous models of the circadian clock. Because CKI represents one of the best drug targets in the circadian regulatory system, understanding if its inhibition speeds up or delays the clock is of central importance. In a collaboration between mathematical modeling and experimental biology, we have tested a detailed mathematical model of the clock and experimentally verified a key and unexpected prediction: The short-period mutations of CKI are in fact gain-of-function, not loss-of-function, mutations. We found that CKI $\varepsilon^{\text {tau }}$ accelerates the degradation of the PER proteins (Gallego et al. 2006b).

\section{THE CORE CLOCK IS A NEGATIVE FEEDBACK LOOP REGULATED BY PROTEIN PHOSPHORYLATION}

The heart of the mammalian clock is a transcriptiontranslation negative feedback loop with a delay between the signal and the negative feedback (Fig. 1) (Lowrey and Takahashi 2004; Schibler and Naef 2005). The oscillations this establishes are then reinforced by additional positive feedback loops. The negative feedback is provided by PERIOD (PER1 and PER2) and CRYPTOCHROME (CRY1 and CRY2) proteins inhibiting the heterodimeric transcription factors CLOCK and BMAL1. CLOCK and BMAL1 drive expression both of circadian output genes and, importantly, their own negative regulators PER1, PER2, CRY1, and CRY2. The abundance of PER1 and PER2 is in turn controlled by protein phosphorylation by

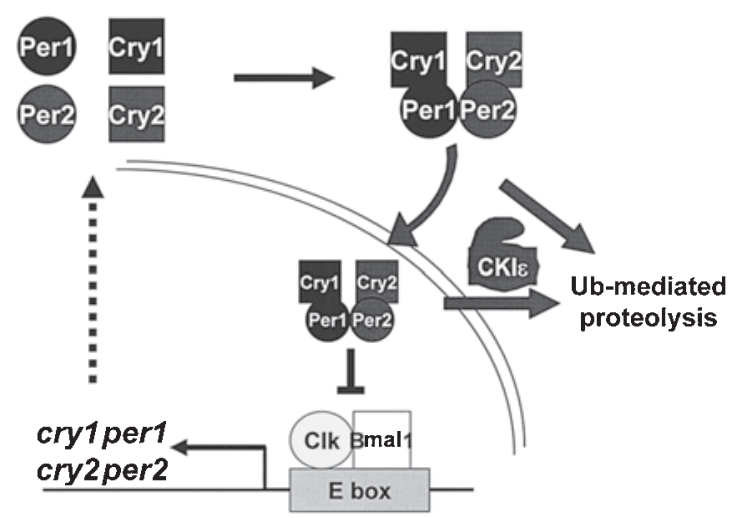

Figure 1. Circadian Rhythms are controlled by a phosphorylation-regulated negative feedback loop. Early in the circadian cycle, PER, CKI, and CRY proteins multimerize in the cytoplasm and then translocate to the nucleus to repress the CLK:BMAL1 transcription factor. Potential functional effects of

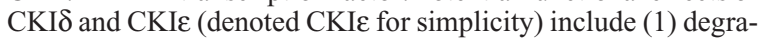
dation of PER early in the accumulation phase, delaying repression; (2) regulating PER nuclear entry of the inhibitor complex, or (3) promoting degradation of PER, thereby terminating repression. The stabilizing Rev-Erb $\alpha$ loop is not shown here.
CKI $\varepsilon$ and CKI $\delta$. In cell-based assays, we have recently reported that CKI $\varepsilon$ and CKI $\delta$ accelerate the proteolysis of PER1 and PER2 by creating a binding site for the ubiquitin ligase $\beta$-TrCP ( $\beta$-transducin repeat-containing protein) (Eide et al. 2005). This is likely to be a key element speeding up the clock, because both CKI inhibitors and proteasome inhibitors slow the clock. However, our result in cell-based systems is in direct conflict with the animalbased result that the tau mutation, shown to decrease CKIE activity in vitro, speeds up the clock. Building on a detailed and predictive quantitative model of circadian rhythms, we have resolved this conflict, providing new insights into basic clock properties (Gallego et al. 2006b).

\section{PHOSPHATASES IN THE CLOCK}

Regulated phosphorylation and degradation of PER is important in controlling the timing of the mammalian clock. Because protein phosphorylation is reversible, protein phosphatases may regulate circadian rhythms as well (Comolli et al. 1996, 2003; Sathyanarayanan et al. 2004; Yang et al. 2004). Protein phosphatase 1 (PP1) and protein phosphatase 2A (PP2A) have recently been implicated in core clock control. Mutations in PP1 and PP2A alter period in lower organisms, and specific PP2A regulators have been shown to regulate PER dynamics and period in Drosophila (Sathyanarayanan et al. 2004; Yang et al. 2004; Schafmeier et al. 2005). Our data show that phosphatases have a role in mammalian circadian rhythm regulation as well and strongly implicate PP1 (Gallego et al. 2006a). Oscillations in PP1 regulators are a potential route to regulating PP1 activity and PER degradation. A robust quantitative model of circadian rhythm will need to incorporate both kinase and phosphatase control of key regulatory steps in the clock.

\section{A PRELIMINARY QUANTITATIVE MODEL OF THE MAMMALIAN CIRCADIAN CLOCK}

How much of the mechanism of the mammalian circadian clock is known? Are the genes, proteins, and molecular interactions, which have been identified as key components in the mammalian circadian clock, sufficient to explain the robust 24-hour timing seen in mammalian cells? To answer these questions, we can convert all of the known genes, proteins, and molecular interactions into a quantitative model and see if this model reproduces the known properties of circadian timing. An initial attempt at modeling the mammalian circadian clock was conducted by Forger and Peskin (2003). Unlike other modeling studies that aim solely at parsimony or arriving at a model which is a good candidate for mathematical analysis, the goal of this work was to represent the available (at the time of the model's development) experimental data on the mammalian circadian clock as accurately as possible.

This model considers the dynamics of the clock proteins PER1, PER2, CRY1, CRY2, Rev-Erbo, CKIE, and $\mathrm{CKI} \delta$ (although these last two kinases are treated as the same). CLK-BMAL1 is assumed to be constitutively bound to the promoters of the relevant genes, based on published experimental data (Kume et al. 1999; 
Yamaguchi et al. 2000; Etchegaray et al. 2003). RevErbo is assumed to form a dimer that can bind to the CRY1 promoter (Preitner et al. 2002). When one or more molecules of the PER/CRY complex (or Rev-Erb $\alpha$ ) are bound to a promoter, transcription ceases (Etchegaray et al. 2003). The only posttranslational modification that the model currently includes is phosphorylation of PER 1 and PER2. When these proteins are phosphorylated, they are targeted for ubiquitin-mediated degradation (Eide et al. 2005; Shirogane et al. 2005). The model assumes that phosphorylation allows the PER proteins to enter the nuclear of the cell (Vielhaber et al. 2000; Akashi et al. 2002). We also assumed that a alternative phosphorylation event blocked the nuclear import of PER1 (but not PER2) to match experimental data (Vielhaber et al. 2000; Yagita et al. 2002).

With these biological assumptions, a quantitative model was developed based on mass action (Forger and Peskin 2003). Variables are the concentrations of the various states of mRNAs and protein complexes and the probability that binding sites on a promoter are bound by transcription factors. This leads to a set of differential equations with multiple rate constants (Forger and Peskin 2003). These rate constants were estimated by choosing those that gave the best predictions of the time courses of the concentrations of clock proteins using a direct search method.

This model has made many important predictions of the effects of circadian clock mutations (Forger and Paydarfar 2004; Forger and Peskin 2004; Locke et al. 2005; Indic and Brown 2006; also see below). The model can also be simulated with the Gillespie Method, which accounts for the stochastic interaction of individual molecules in a cell (Forger and Peskin 2005). Using this method, predictions can be made about the accuracy of timing in the wild-type mammalian circadian clock and in the presence of mutations of clock genes. Although this model will need to be regularly updated reflecting both recent data and the results from experiments in this proposal, it presents a powerful tool for understanding clock function and interpreting experimental data.

\section{EXPERIMENTAL ADVANCES}

Our initial studies demonstrated that phosphorylation controlled the nuclear localization of mPER1, and we mapped phosphorylation-dependent nuclear localization sequences and nuclear export signals (Vielhaber et al. 2000). Novel nuclear export sequences were discovered in PER1 and PER2 that enable the PER proteins to be exported from the nucleus as well (Vielhaber et al. 2001). Because CKI $\varepsilon$ is in a multiprotein complex with multiple circadian proteins, we asked if other circadian regulators were targets of CKIE. We found that CRY1 and CRY2 are phosphorylated by CKIE but only when they bind to a PER/CKIE complex. BMAL1 is also an in vivo CKIe substrate. Knock down of CKIE by RNA interference (RNAi), expression of a dominant-negative CKIع (K38A), or use of a CKI inhibitor lead to decreased phosphorylation of BMAL1 in cells and decreased transcriptional activation activity of BMAL1:CLOCK (Eide et al. 2002; Gallego et al. 2006b).

\section{PER2 PROTEIN STABILITY IS CONTROLLED BY PROTEIN PHOSPHORYLATION AND UBIQUITIN-MEDIATED PROTEASOMAL DEGRADATION}

We and other investigators found that although PER1 nuclear localization is regulated by CKIE, PER2 localization is not altered by CKI $\varepsilon$ or CKI $\delta$ expression (Vielhaber et al. 2000; Akashi et al. 2002; Takano et al. 2004). This suggests that phosphorylation of PER2 has distinct regulatory effects. We next reported that CKI-regulated phosphorylation of PER2 changes its stability (Eide et al. 2005; Gallego et al. 2006b). The quantitative model indicates that control of PER2 abundance is likely to be a major control point in proper clock timing (Gallego et al. 2006b).

As we investigated how protein phosphorylation is a critical regulator of PER2 stability, we found that both kinases and protein phosphatases are important in this control (Fig. 2) (Gallego and Virshup 2007). Treatment of cells expressing full-length PER2 with a cell-permeable serine/threonine phosphatase inhibitor (either okadaic acid or calyculin A) causes rapid degradation of PER2 (Eide et al. 2005). Degradation requires the activity of CKI, because pretreatment of cells with the CKI inhibitor IC261 blocks PER2 degradation. PER2 degradation is ubiquitinmediated and proteasome-dependent, because pretreatment with the proteasome inhibitor MG-132 blocks mPER2 degradation and causes the accumulation of polyubiquitinated PER2. An important cellular ubiquitin E3 ligase, the stem cell factor (SCF), recognizes phosphorylated proteins before their degradation. The substraterecognition protein in this complex is the adapter protein $\beta$-TrCP (see model in Fig. 2). We showed that a dominantnegative form of $\beta$-TrCP (able to interact with phosphorylated substrates but lacking the "F box" and unable to interact with the remainder of the SCF) is able to bind to phosphorylated PER2 and block both its ubiquitination and proteolysis (Eide et al. 2005). This result was subsequently confirmed by others (Shirogane et al. 2005). We have identified the phosphatase that regulates PER2 phosphorylation stability as PP1 (Gallego et al. 2006a).

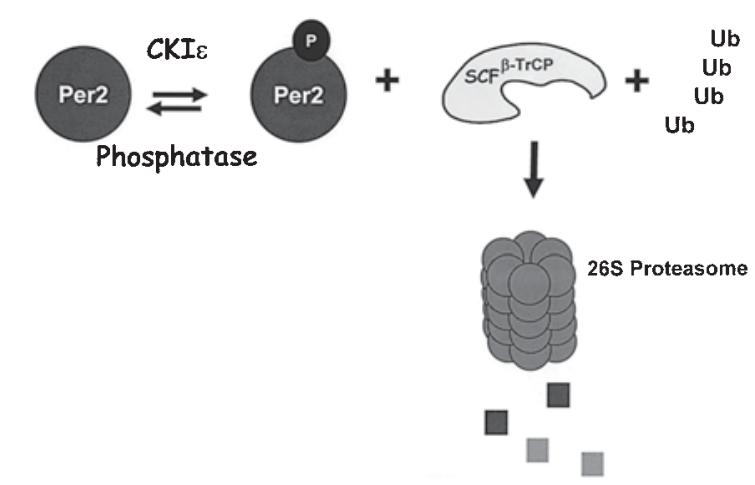

Figure 2. Phosphorylation controls the abundance of PER2. Phosphorylation of a specific site on PER2 is regulated by the balance of CKI and phosphatase activity. $\mathrm{SCF}^{\beta-\operatorname{TrCP}}$ binds to phosphorylated PER2 and drives ubiquitinylation and subsequent proteasome-dependent proteolysis. Destruction of PER2 will relieve inhibition of the CLK:BMAL1 complex shown in Fig. 1. 
Studying CKIE-regulated PER2 stability, we found that $\beta$-TrCP interacts with PER2 via the sequence ${ }^{477}$ SSGYGS $^{482}$, similar to the consensus $\beta$-TrCP recognition motif DSG $\phi X S$, where $\phi$ is hydrophobic (Wu et al. 2003). Mutation to ${ }^{477}$ ASAYGS ${ }^{482}$ (mPER2 [S477A/ G479A]) abolished almost all detectable CKIE-dependent $\beta$-TrCP binding to PER2 and inhibited PER2 degradation (Eide et al. 2005). The simplest model from these data is that CKIE phosphorylation of PER2 at Ser-478 and Ser482 creates a $\beta$-TrCP-binding site leading to ubiquitination and degradation of PER2.

\section{A CELL-BASED ASSAY FOR CIRCADIAN RHYTHMS}

A key step in the analysis of circadian rhythms is to test if biochemical phenomenon dissected in vitro and in cells actually affects clock function. Until recently, this has relied heavily on analysis of rhythms in whole animals. However, the observation that individual cells throughout the body have an intrinsic clock and that these clocks can be synchronized in cultured cells (Balsalobre et al. 1998; Yamazaki et al. 2000) has enabled a new generation of assays examining circadian rhythms. The initial method described by the Schibler lab assessed circadian gene expression by ribonuclease protection assay from cells harvested every 3 hours for 4 days (Balsalobre et al. 1998). We postulated that a similar cell-culture-based system with a real-time luciferase reporter would be a more robust and less labor-intensive method to measure circadian rhythms. Using $6.7 \mathrm{~kb}$ of the $m P E R 1$ promoter driving luciferase (Yamazaki et al. 2000), stable Rat-1a cell transfectants were developed that display seruminducible and rhythmic luciferase activity; these cells are denoted Rat-1 (Per1::luc). To follow circadian rhythms in real time, cells are incubated in medium supplemented with luciferin (described in Eide et al. 2005). The primary light-output data are collected in a 24-well luminometer and smoothed by subtracting the 24-hour running mean. Rat-1 (Per $1: \because l u c$ ) cells show sustained and consistent circadian oscillations of luciferase abundance (Fig. 3). The period, phase, and amplitude are then quantitatively determined by Fourier transform of the data. The end result is a useful system that allows us to directly test both model predictions and molecular hypotheses (Eide et al. 2005).

\section{THE PROTEASOME REGULATES MAMMALIAN CIRCADIAN RHYTHM}

We tested two hypotheses about the effect of proteasome inhibition on clock activity. If this accelerated accumulation of PER2 early in the cycle, it might speed up the clock, whereas if it delayed degradation late in the cycle, it might prolong repression of CLOCK/BMAL1 and slow the clock. We found that proteasome inhibition caused a significant lengthening of period. This result suggests, as predicted by the quantitative model, that degradation of PER proteins is a key event shortening circadian period; therefore, inhibition of PER degradation lengthens period (see model in Fig. 1). However, a number of circadian regulators including both PER proteins are degraded by

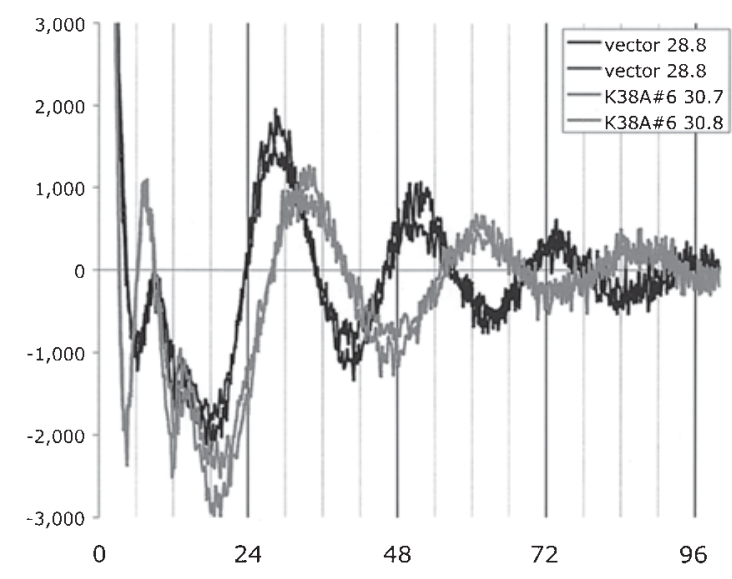

Figure 3. Genetic manipulation of circadian rhythm in cultured cells. Dominant-negative CKI $\varepsilon$ slows down the clock. Rat-1 (per $1: \because l u c$ ) cells were transduced with retrovirus expressing CKIE (K38A) (light gray) or an empty vector (black). Independent isolates gave similar results. The DbtS P47S mutation engineered into CKIE shortens period. Two independent isolates (light gray) both showed period shortening.

the proteasome and could be altered by proteasome inhibition. It remains critical to specifically test whether specific inhibition of PER2 degradation lengthens the period. The mutations in the F-box protein Fbxl3 indicate that impaired CRY protein degradation also lengthens period (Busino et al. 2007; Godinho et al. 2007; Siepka et al. 2007; Virshup and Forger 2007).

\section{INHIBITION OF CKIE UNEXPECTEDLY LENGTHENS CIRCADIAN PERIOD}

To further test the hypothesis that CKI\& phosphorylation of PER targets it for proteasomal degradation, the effect of the $\mathrm{CKI} / \varepsilon$ inhibitor IC261 was assessed. Using our newly developed cell-based system, we found the surprising result that inhibition of CKI both delayed PER degradation and lengthened the circadian period in cells (Eide et al. 2005). Similar results have now been reported for another CKI inhibitor in intact animals (Badura et al. 2007).

The tau mutant hamster has been instrumental in developing models of the circadian clock. First reported in 1988 , the tau heterozygote has a period of 22 hours, whereas the homozygous mutant has a period of 20 hours (Ralph and Menaker 1988). The cause of the short period is a point mutation in hamster CKIE that decreases in vitro kinase activity (Lowrey et al. 2000). Subsequently, we contributed to the description of a human family with a short circadian period with a serine $\rightarrow$ glycine mutation in PER2 in the CKIc-binding domain (Jones et al. 1999; Toh et al. 2001). These two mutations strongly suggest that phosphorylation of PER2 by CKI is important for proper period length. Importantly, they predicted that decreased CKIE phosphorylation of PER2 should shorten mammalian period. However, our experiments described here indicate that this is not the case. Unlike the results expected from the tau hamster with low CKIE activity, inhibition of CKI in cells lengthens period.

One concern is that the effects of proteasome and 


\section{PHOSPHORYLATION REGULATES THE CLOCK}

kinase inhibitors might be nonspecific. We therefore inhibited CKIE activity in cells by expression of a dominant-negative CKIع. We used a retrovirus driving expression of wild-type CKIE or dominant-negative CKIE (K38A) in our Rat-1 reporter cells. As Figure 3 shows, overexpression of dominant-negative CKI\& lengthens the period. Ribonuclease protection assays showed that this effect was not limited to the perl promoter but affected the timing of expression of several circadian output genes (data not shown). This is consistent with the results of our inhibitor assay and indicates that the critical effect of CKIE is to shorten the period (hence, inhibition lengthens period). On the basis of model predictions, we postulated that shortening of the period is due to the ability of CKI to accelerate degradation of PER proteins.

Do all mutants of CKI that shorten period increase CKI activity in vivo? As a first step to testing this, we made Rat-1 (Per $1:: l u c$ ) cell lines expressing CKIE with the Drosophila doubletime short (DbtS) (P47S) mutation. The mutant proline 47 residue is highly conserved in the CKI family and the $D b t S$ allele has the strongest effect of any period-shortening mutant (Kloss et al. 1998; Price et al. 1998). Like the K38A mutation, the $\mathrm{DbtS}$ mutation decreases $\mathrm{CKI} \varepsilon$ kinase activity in vitro (Eide and Virshup 2001; Preuss et al. 2004). Importantly, although expression of the truly kinase-inactive CKIE (K38A) lengthens period, CKIE $(D b t S)$ expression shortens period, similar to the effect it has in Drosophila (Fig. 3). Importantly, the different effects of distinct CKIE alleles on period in cell lines mirror the results in mutant animals. These data indicate that the Rat-1 (Per1::luc) cells provide an experimentally tractable model system to study the biochemical basis for the ability of CKIE to alter rhythm.

\section{MATHEMATICAL MODELING PREDICTS AN OPPOSITE PHENOTYPE FOR TAU}

Our experimental data obtained in Rat-1 (Per1::luc) cells treated with kinase and proteasome inhibitors or dominant-negative CKIE agree with the predictions of the quantitative model of the circadian molecular clock (Forger and Peskin 2003), arguing that the CKI $\varepsilon^{\text {tau }}$ mutant must be a gain of function, not a loss of function. Recall, however, that $\mathrm{CKI} \varepsilon^{\text {tau }}$ has an eightfold decrease in activity in vitro (Lowrey et al. 2000; Preuss et al. 2004). Previously, several potential conceptual models had attempted to explain how a decrease in function of $\mathrm{CKI} \delta / \varepsilon$ might cause the fast period phenotype of the tau mutation. However, when we decreased the rate of phosphorylation of PER1 or PER2 in the Forger-Peskin model, we consistently found that it predicted a longer, not a shorter, period: A $50 \%$ decrease in the rate of the primary phosphorylation of PER1 and PER2 lengthened the period of the model by 0.13 and 2.43 hours, respectively. Remarkably, in both cases, the Forger-Peskin model predicts that changes in CKI activity on PER2 stability have a far greater impact than changes in localization or in phosphorylation of PER1. None of the previously proposed explanations of tau are consistent with the quantitative model's predictions. In the detailed and otherwise accurate mathematical model of the clock, only increased kinase activity resulting in increased PER2 degradation was able to produce significant short-period phenotypes.

\section{AN OPPOSITE ROLE FOR TAU}

If the tau mutation in fact increased, rather than decreased, the phosphorylation and degradation of the PER proteins, a shorter period would be expected and this conflict would be resolved. It would also show that the model was both correct and useful. We therefore tested this model prediction-that CKI $\varepsilon^{\text {tau }}$ increased PER2 degradation - directly in cell-based assays (Fig. 4, right). The half-life of PER2 was measured in the presence of wild-type CKIE, CKI $\varepsilon^{\text {tau }}$, or CKIع (K38A) (a kinase-inactive mutant). Although expression of wild-type CKIE modestly shortened PER2 half-life, CKI ${ }^{\text {tau }}$ expression markedly decreased PER2 half-life. Similar results were seen for PER1 (Gallego et al. 2006b). CKIE (K38A) had no significant effect on PER abundance and half-life,
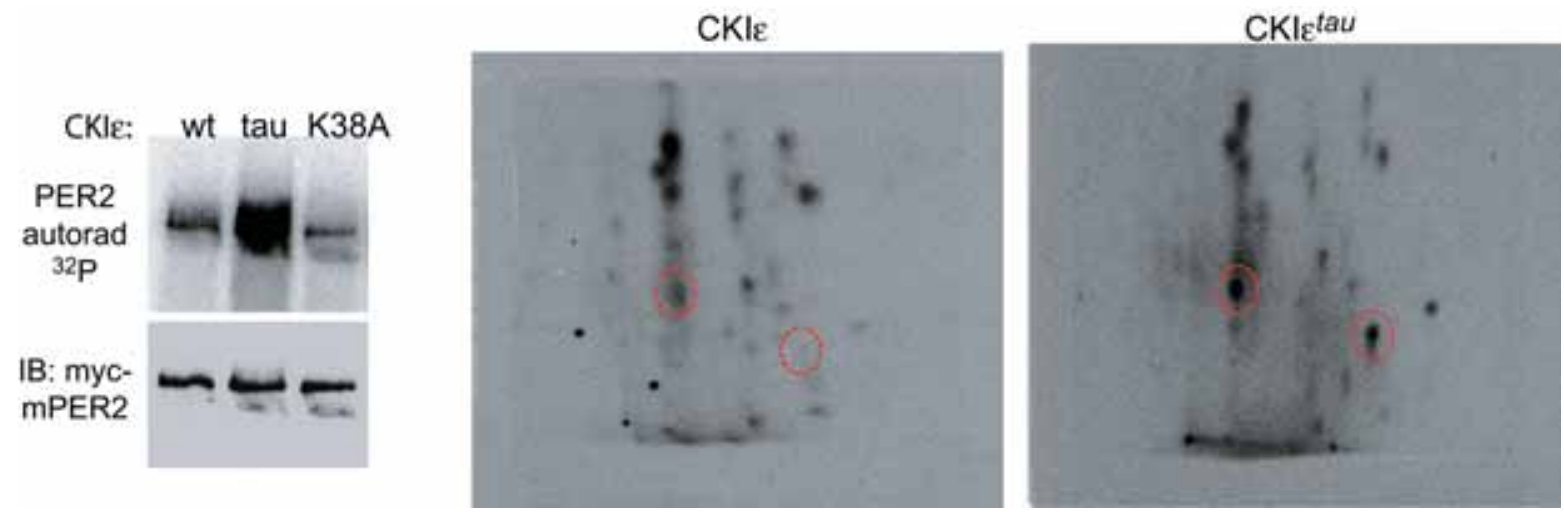

Figure 4. $t a u$ is a gain-of-function mutation in vivo that stimulates the site-specific phosphorylation of PER2. (Left) CKI $\varepsilon^{\text {tau }}$ expression increases the in vivo phosphorylation of PER2. PER2 was coexpressed with the indicated form of CKIE and cells were metabolically labeled with ${ }^{32} \mathrm{P}$ orthophosphate for 4 hours in the presence of MG132. myc-tagged PER2 was then immunoprecipitated, followed by autoradiography and immunoblotting. (Right) CKI $\varepsilon^{\text {tau }}$ increases phosphorylation of two specific peptides. The metabolically labeled PER2 was analyzed by trypsin/chymotrypsin digestion and two-dimensional phosphopeptide mapping. There may be two motifs whose phosphorylation is increased by CKI $\varepsilon^{\text {tau }}$, or the protease digestion may cleave a single motif into half, creating two phosphopeptides with increased labeling. 
notably distinct from the effect of CKI $\varepsilon^{\text {tau }}$. These results indicate that in cells, the tau mutation is a gain of function that leads to the more rapid degradation of PER 1 and PER2. This CKI $\varepsilon^{\text {tau }}$-accelerated degradation still requires the $\beta$ - TrCP/proteasome pathway, because it is inhibited by MG132, and a dominant-negative form of $\beta$-TrCP.

Because CKI $\varepsilon^{\text {tau }}$ causes the rapid degradation of PER2, it should be a gain-of-function mutation. This conflicts with reports that $\mathrm{CKI} \varepsilon^{\text {tau }}$ has decreased kinase activity in vitro (Lowrey et al. 2000). Our data show that the tau mutation accelerates the degradation of PER1 and PER2 via the ubiquitin/proteasome pathway. We then tested the prediction of the Forger-Peskin model that CKI $\varepsilon^{\text {tau }}$ phosphorylated PER more efficiently than wild-type kinase in cells, rather than in vitro. Wild-type CKI $\varepsilon$ expression in cultured cells caused a small increase in both PER1 and PER2 phosphorylation, whereas the catalytically inactive CKIE (K38A) had little effect. Notably, in vivo, CKI $\varepsilon^{\text {tau }}$ caused markedly increased phosphorylation of PER proteins compared with wild-type kinase (Fig. 4, left). This is consistent with CKIE $\varepsilon^{\text {tau }}$ being a gain-of-function mutation in vivo (although not in vitro) specific to the PER proteins.

Importantly, we found that $\mathrm{CKI} \varepsilon^{\text {tau }}$ showed decreased activity on most substrates. We considered two potential mechanisms for the specific increased in vivo phosphorylation of PER2. CKI $\varepsilon^{\text {tau }}$ may selectively phosphorylate a site in PER that specifically regulates its degradation or CKI $\varepsilon^{\text {tau }}$ may globally increase phosphorylation of PER in vivo despite its decreased activity in vitro. To differentiate between these possibilities, in vivo metabolically ${ }^{32} \mathrm{P}$ labeled PER2 (Fig. 4, right) and PER1 (not shown) were examined by two-dimensional phosphopeptide mapping (Firulli et al. 2004). Expression of wild-type CKIE did not lead to any apparent changes in the pattern of PER phosphorylation, indicating that endogenous CKIE is not ratelimiting for PER phosphorylation within the cells. Coexpression of CKI $\varepsilon^{\text {tau }}$ caused a marked increase in phosphorylation of two PER2 peptides. This result has been reproduced in four independent experiments. This suggests that two specific peptides within PER2 are phosphorylated much more efficiently by $\mathrm{CKI} \varepsilon^{\mathrm{tau}}$ than by wild-type CKIع.

We have preliminarily identified the specific phosphorylation sites controlling the stability of PER2. We first identified a CKI phosphorylation-dependent $\beta$-TrCPbinding site in PER2 (amino acids 477-482) (Eide et al. 2005). Enhanced CKI $\varepsilon^{\text {tau }}$ phosphorylation in this motif may promote PER2 degradation. To test this, we compared the effect of $\mathrm{CKI}^{\mathrm{tau}}$ expression on wild-type and S477A G479A PER2 that cannot bind $\beta$-TrCP (Eide et al. 2005). Mutation of these sites blocked CKI $\varepsilon^{\text {tau }}$-induced degradation. This suggests the specific hypothesis that CKI $\varepsilon^{\text {tau }}$ has increased activity on the PER $2 \beta$-TrCP-binding motif, thereby creating a $\beta$-TrCP-binding site and accelerating PER2 degradation.

\section{PROTEIN PHOSPHATASES REGULATE MPER2 DEGRADATION AND CIRCADIAN RHYTHM}

The ability of cell-permeable phosphatase inhibitors okadaic acid and calyculin A to cause PER2 degradation suggested that cellular protein phosphatases can also reg- ulate PER2 stability and hence circadian rhythms (Eide et al. 2005). Okadaic acid and calyculin A inhibit both PP1 and PP2A phosphatases. As an initial test to discriminate between PP1 and PP2A, we examined the stability of invitro-translated ${ }^{35} \mathrm{~S}$-labeled mPER2 in cell extracts in the presence of phosphatase inhibitors.

We found that PER2 was phosphorylated but not degraded over 3 hours in cell extracts, although addition of the specific PP1 inhibitor, inhibitor-2, caused a rapid degradation of the protein. This suggested that PER2 is a specific substrate of PP1, and PP1 can regulate the phosphorylation and degradation of PER2. PER2 can be dephosphorylated by PP1 immunoprecipitated from mouse brain, liver, and Rat-1a cells (Gallego et al. 2006a). To examine if PER2 and PP1 interact, PP1 and PER2 were coexpressed in HEK 293 cells. Immunoprecipitation of PP1 brought down PER2 and immunoprecipitation of PER2 brought down PP1. Thus, PP1 can interact with and dephosphorylate PER2.

If PP1 controls PER2 stability, then we postulate that a dominant-negative PP1 would enhance PER2 phosphorylation and accelerate its degradation. We generated a dominant-negative PP1 (D95N) and coexpressed it with PER2. Expression of dominant-negative but not wildtype green fluorescent protein (GFP)-tagged PP1 caused a marked decrease in PER2 abundance. This decrease can be blocked by dominant-negative $\beta$-TrCP and proteasome inhibitors, consistent with the hypothesis that PP1 can regulate phosphorylation and hence stability of PER2 (Gallego et al. 2006a).

Both PP1 and PP2A are regulated by tightly associated targeting/regulatory proteins (Gallego and Virshup 2005). Sehgal and coworkers (Sathyanarayanan et al. 2004) reported that in Drosophila, PP2A targeting proteins could regulate dPER stability during circadian rhythms, and they presented genetic evidence that PP2A was a clock regulator. We therefore examined synchronized Rat- 1 cells, as well as mouse livers, for evidence of oscillations in both PP1 and PP2A targeting/regulatory proteins. We have detected significant circadian oscillation of PP1-binding proteins in both Rat-1 fibroblasts (Fig. 5, center) and mouse liver (Fig. 5, left). Immunoblots examining PP2A regulators have shown no circadian oscillation of any of these proteins (Fig. 5, right). The data, taken together, are consistent with the hypothesis that PP1 and an oscillating PP1-targeting protein regulate the phosphorylation and stability of PER 2 in the mammalian circadian clock.

\section{CONCLUSIONS}

Reversible phosphorylation and regulated degradation of key circadian regulators have an important role in determining circadian timing. The degradation of PERIOD, and now CRYPTOCHROMES, in mammalian cells is clearly important, because perturbations in those rates have highly significant effects on the length of a cycle. The evidence to date indicate that both CKI $\delta$ and CKIE are important in the clock, although it is not clear if they have overlapping, identical, or distinct roles. Regulated destruction of PER protein is determined by a 


\section{PHOSPHORYLATION REGULATES THE CLOCK}
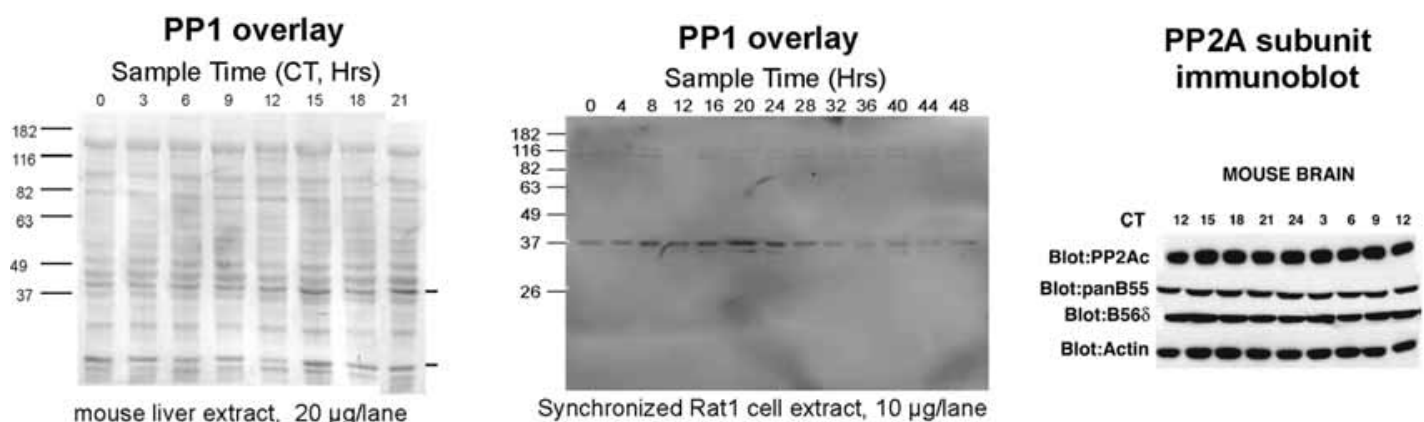

Figure 5. PP1 regulators vary during the circadian cycle. (Left and center panels) PP1 overlay detects PP1-binding proteins. Livers (left) and brains (far right) from mice kept in 12 hours light:12 hours dark. (Middle) Rat-1 cells synchronized with forskolin pulse were lysed at the indicated times. All samples were separated by SDS-PAGE and transferred to PVDF membranes. (Left and center) Membranes were incubated with the digoxygenin-labeled PP1 catalytic subunit (Beullens et al. 1999), and PP1-binding proteins were detected with antidigoxygenin antibodies (the overlay was performed in collaboration with the S. Shenolikar lab at Duke University). (Right panel) No evidence for circadian oscillations of PP2A regulators. Brain and liver (not shown) extracts were probed with antibodies to the PP2A regulatory subunits PP2Ac, B56 $\delta$, and a pan-B55 (PR55), as well as B56 $\beta, \delta$, and PR72 (not shown). No circadian variation was detected for any PP2A subunit in either brain or liver.

balance of CKI activity and protein phosphatase activity. There is a role for PP2A in regulating protein stability of PER in Drosophila, and we found a role for mammalian cells. The evidence from a number of laboratories that the rhythmic expression of PER and perhaps CRY is not essential to maintaining a rhythm supports a somewhat heretical hypothesis that rhythmic destruction of the regulators is the most numerically important regulator of the core clock.

\section{ACKNOWLEDGMENTS}

These studies were funded in part by R01GM060387 and the Huntsman Cancer Foundation.

\section{REFERENCES}

Akashi M., Tsuchiya Y., Yoshino T., and Nishida E. 2002. Control of intracellular dynamics of mammalian period proteins by casein kinase I epsilon (CKIepsilon) and CKIdelta in cultured cells. Mol. Cell. Biol. 22: 1693.

Antoch M.P., Kondratov R.V., and Takahashi J.S. 2005. Circadian clock genes as modulators of sensitivity to genotoxic stress. Cell Cycle 4: 901.

Badura L., Swanson T., Adamowicz W., Adams J., Cianfrogna J., Fisher K., Holland J., Kleiman R., Nelson F., Reynolds L., St. Germain K., Schaeffer E., Tate B., and Sprouse J. 2007. An inhibitor of casein kinase I $\varepsilon$ induces phase delays in circadian rhythms under free-running and entrained conditions. $J$. Pharmacol. Exp. Ther. 322: 730 .

Balsalobre A., Damiola F., and Schibler U. 1998. A serum shock induces circadian gene expression in mammalian tissue culture cells. Cell 93: 929

Beullens M., Van Eynde A., Vulsteke V., Connor J., Shenolikar S., Stalmans W., and Bollen M. 1999. Molecular determinants of nuclear protein phosphatase-1 regulation by NIPP-1. $J$. Biol. Chem. 274: 14053.

Busino L., Bassermann F., Maiolica A., Lee C., Nolan P.M., Godinho S.I., Draetta G.F., and Pagano M. 2007. SCFFbxl3 controls the oscillation of the circadian clock by directing the degradation of cryptochrome proteins. Science 316: 900.

Chang D.C. and Reppert S.M. 2001. The circadian clocks of mice and men. Neuron 29: 555.

Comolli J.C., Fagan T., and Hastings J.W. 2003. A type-1 phosphoprotein phosphatase from a dinoflagellate as a possible component of the circadian mechanism. J. Biol. Rhythms 18: 367.
Comolli J., Taylor W., Rehman J., and Hastings J.W. 1996. Inhibitors of serine/threonine phosphoprotein phosphatases alter circadian properties in Gonyaulax polyedra. Plant Physiol. 111: 285.

Eide E.J. and Virshup D.M. 2001. Casein kinase I: Another cog in the circadian clockworks. Chronobiol. Int. 18: 389.

Eide E.J., Vielhaber E.L., Hinz W.A., and Virshup D.M. 2002. The circadian regulatory proteins BMAL1 and cryptochromes are substrates of casein kinase IE (CKIE). J. Biol. Chem. 277: 17248 .

Eide E., Woolf M., Kang H., Woolf P., Camacho F., Vielhaber E., Giovanni A., and Virshup D. 2005. Control of mammalian circadian rhythm by CKIE-regulated proteasome-mediated PER2 degradation. Mol. Cell. Biol. 25: 2795.

Etchegaray J.P., Lee C., Wade P.A., and Reppert S.M. 2003. Rhythmic histone acetylation underlies transcription in the mammalian circadian clock. Nature 421: 177.

Firulli B.A., Virshup D.M., and Firulli A.B. 2004. Phosphopeptide mapping of proteins ectopically expressed in tissue culture cell lines. Biol. Proced. Online 6: 16.

Forger D.B. and Paydarfar D. 2004. Starting, stopping, and resetting biological oscillators: In search of optimum perturbations. J. Theor. Biol. 230: 521.

Forger D.B. and Peskin C.S. 2003. A detailed predictive model of the mammalian circadian clock. Proc. Natl. Acad. Sci. 100: 14806.

. 2004. Model based conjectures on mammalian clock controversies. J. Theor. Biol. 230: 533.

2005. Stochastic simulation of the mammalian circadian clock. Proc. Natl. Acad. Sci. 102: 321

Fu L., Pelicano H., Liu J., Huang P., and Lee C. 2002. The circadian gene Period2 plays an important role in tumor suppression and DNA damage response in vivo. Cell 111: 41.

Gallego M. and Virshup D.M. 2005. Protein serine/threonine phosphatases: Life, death, and sleeping. Curr. Opin. Cell Biol. 17: 197.

2007. Post-translational modifications regulate the ticking of the circadian clock. Nat. Rev. Mol. Cell Biol. 8: 139

Gallego M., Kang H., and Virshup D.M. 2006a. Protein phosphatase 1 regulates the stability of the circadian protein PER2. Biochem. J. 399: 169

Gallego M., Eide E., Woolf M., Virshup D., and Forger D. 2006b. An opposite role for tau in circadian rhythms revealed by mathematical modeling. Proc. Natl. Acad. Sci. 103: 10618.

Godinho S.I., Maywood E.S., Shaw L., Tucci V., Barnard A.R., Busino L., Pagano M., Kendall R., Quwailid M.M., Romero M.R., O'Neill J., Chesham J.E., Brooker D., Lalanne Z., Hastings M.H., and Nolan P.M. 2007. The after-hours mutant reveals a role for Fbx13 in determining mammalian circadian period. Science 316: 897. 
Indic P. and Brown E.N. 2006. Characterizing the amplitude dynamics of the human core-temperature circadian rhythm using a stochastic-dynamic model. J. Theor. Biol. 239: 499.

Jones C.R., Campbell S.S., Zone S.E., Cooper F., DeSano A., Murphy P.J., Jones B., Czajkowski L., and Ptacek L.J. 1999. Familial advanced sleep-phase syndrome: A short-period circadian rhythm variant in humans. Nat. Med. 5: 1062.

Kloss B., Price J.L., Saez L., Blau J., Rothenfluh A., Wesley C.S., and Young M.W. 1998. The Drosophila clock gene double-time encodes a protein closely related to human casein kinase I epsilon. Cell 94: 97.

Kume K., Zylka M.J., Sriram S., Shearman L.P., Weaver D.R., Jin X., Maywood E.S., Hastings M.H., and Reppert S.M. 1999. mCRY1 and mCRY2 are essential components of the negative limb of the circadian clock feedback loop. Cell 98: 193.

Locke J.C., Millar A.J., and Turner M.S. 2005. Modelling genetic networks with noisy and varied experimental data: the circadian clock in Arabidopsis thaliana. J. Theor. Biol. 234: 383.

Lowrey P.L. and Takahashi J.S. 2004. Mammalian circadian biology: Elucidating genome-wide levels of temporal organization. Annu. Rev. Genomics Hum. Genet. 5: 407.

Lowrey P.L., Shimomura K., Antoch M.P., Yamazaki S., Zemenides P.D., Ralph M.R., Menaker M., and Takahashi J.S. 2000. Positional syntenic cloning and functional characterization of the mammalian circadian mutation tau. Science 288: 483 .

Mansour H.A., Monk T.H., and Nimgaonkar V.L. 2005. Circadian genes and bipolar disorder. Ann. Med. 37: 196.

Matsuo T., Yamaguchi S., Mitsui S., Emi A., Shimoda F., and Okamura H. 2003. Control mechanism of the circadian clock for timing of cell division in vivo. Science 302: 255 .

Preitner N., Damiola F., Lopez-Molina L., Zakany J., Duboule D., Albrecht U., and Schibler U. 2002. The orphan nuclear receptor REV-ERBalpha controls circadian transcription within the positive limb of the mammalian circadian oscillator. Cell 110: 251

Preuss F., Fan J.Y., Kalive M., Bao S., Schuenemann E., Bjes E.S., and Price J.L. 2004. Drosophila doubletime mutations which either shorten or lengthen the period of circadian rhythms decrease the protein kinase activity of casein kinase I. Mol. Cell. Biol. 24: 886.

Price J.L., Blau J., Rothenfluh A., Abodeely M., Kloss B., and Young M.W. 1998. double-time is a novel Drosophila clock gene that regulates PERIOD protein accumulation. Cell 94: 83.

Ralph M.R. and Menaker M. 1988. A mutation of the circadian system in golden hamsters. Science 241: 1225.

Reppert S.M. and Weaver D.R. 2002. Coordination of circadian timing in mammals. Nature 418: 935.

Rivard G.E., Infante-Rivard C., Dresse M.F., Leclerc J.M., and Champagne J. 1993. Circadian time-dependent response of childhood lymphoblastic leukemia to chemotherapy: A longterm follow-up study of survival. Chronobiol. Int. 10: 201.

Sathyanarayanan S., Zheng X., Xiao R., and Sehgal A. 2004. Posttranslational regulation of Drosophila PERIOD protein by protein phosphatase 2A. Cell 116: 603 .

Schafmeier T., Haase A., Kaldi K., Scholz J., Fuchs M., and Brunner M. 2005. Transcriptional feedback of Neurospora circadian clock gene by phosphorylation-dependent inactivation of its transcription factor. Cell 122: 235.

Schibler U. and Naef F. 2005. Cellular oscillators: Rhythmic gene expression and metabolism. Curr. Opin. Cell Biol. 17: 223.

Schmiegelow K., Glomstein A., Kristinsson J., Salmi T.,
Schroder H., and Bjork O. 1997. Impact of morning versus evening schedule for oral methotrexate and 6-mercaptopurine on relapse risk for children with acute lymphoblastic leukemia (Nordic Society for Pediatric Hematology and Oncology [NOPHO]). J. Pediatr. Hematol. Oncol. 19: 102.

Shirogane T., Jin J., Ang X.L., and Harper J.W. 2005. SCFbetaTRCP controls clock-dependent transcription via casein kinase 1-dependent degradation of the mammalian period-1 (Per1) protein. J. Biol. Chem. 280: 26863.

Siepka S.M., Yoo S.H., Park J., Song W., Kumar V., Hu Y., Lee C., and Takahashi J.S. 2007. Circadian mutant Overtime reveals F-box protein FBXL3 regulation of cryptochrome and Period gene expression. Cell 129: 1011

Suri V., Hall J.C., and Rosbash M. 2000. Two novel doubletime mutants alter circadian properties and eliminate the delay between RNA and protein in Drosophila. J. Neurosci. 20: 7547.

Takano A., Isojima Y., and Nagai K. 2004. Identification of mPer1 phosphorylation sites responsible for the nuclear entry. J. Biol. Chem. 279: 32578 .

Toh K.L., Jones C.R., He Y., Eide E.J., Hinz W.A., Virshup D.M., Ptacek L.J., and Fu Y.H. 2001. An hPer2 phosphorylation site mutation in familial advanced sleep-phase syndrome. Science 291: 1040.

Turek F.W., Joshu C., Kohsaka A., Lin E., Ivanova G., McDearmon E., Laposky A., Losee-Olson S., Easton A., Jensen D.R., Eckel R.H., Takahashi J.S., and Bass J. 2005. Obesity and metabolic syndrome in circadian Clock mutant mice. Science 308: 1043.

Vielhaber E.L., Duricka D., Ullman K.S., and Virshup D.M. 2001. Nuclear export of mammalian PERIOD proteins. $J$. Biol. Chem. 276: 45921.

Vielhaber E., Eide E., Rivers A., Gao Z.-H., and Virshup D.M. 2000. Nuclear entry of the circadian regulator mPER1 is controlled by casein kinase I $\varepsilon$. Mol. Cell. Biol. 20: 4888.

Virshup D.M. and Forger D.B. 2007. After hours keeps clock researchers CRYing Overtime. Cell 129: 857.

Wallace A., Chinn D., and Rubin G. 2003. Taking simvastatin in the morning compared with in the evening: Randomised controlled trial. Br. Med. J. (BMJ) 327: 788.

Wu G., Xu G., Schulman B.A., Jeffrey P.D., Harper J.W., and Pavletich N.P. 2003. Structure of a beta-TrCP1-Skp1-betacatenin complex: Destruction motif binding and lysine specificity of the SCF(beta-TrCP1) ubiquitin ligase. Mol. Cell 11: 1445.

Xu Y., Padiath Q.S., Shapiro R.E., Jones C.R., Wu S.C., Saigoh N., Saigoh K., Ptacek L.J., and Fu Y.H. 2005. Functional consequences of a CKIdelta mutation causing familial advanced sleep phase syndrome. Nature 434: 640 .

Yagita K., Tamanini F., Yasuda M., Hoeijmakers J.H., van der Horst G.T., and Okamura H. 2002. Nucleocytoplasmic shuttling and mCRY-dependent inhibition of ubiquitylation of the mPER2 clock protein. EMBO J. 21: 1301.

Yamaguchi S., Mitsui S., Miyake S., Yan L., Onishi H., Yagita K., Suzuki M., Shibata S., Kobayashi M., and Okamura H. 2000. The $5^{\prime}$ upstream region of mPerl gene contains two promoters and is responsible for circadian oscillation. Curr. Biol. 10: 873.

Yamazaki S., Numano R., Abe M., Hida A., Takahashi R., Ueda M., Block G.D., Sakaki Y., Menaker M., and Tei H. 2000. Resetting central and peripheral circadian oscillators in transgenic rats. Science 288: 682 .

Yang Y., He Q., Cheng P., Wrage P., Yarden O., and Liu Y. 2004. Distinct roles for PP1 and PP2A in the Neurospora circadian clock. Genes Dev. 18: 255. 


\section{$\overbrace{\text { CSH' }}^{\infty}$ Cold Spring Harbor Symposia SYMPOSIA on Quantitative Biology}

\section{Reversible Protein Phosphorylation Regulates Circadian Rhythms}

D. M. Virshup, E. J. Eide, D. B. Forger, et al.

Cold Spring Harb Symp Quant Biol 2007 72: 413-420

Access the most recent version at doi:10.1101/sqb.2007.72.048

References This article cites 59 articles, 26 of which can be accessed free at: http://symposium.cshlp.org/content/72/413.full.html\#ref-list-1

\section{License} Email Alerting $\begin{aligned} & \text { Receive free email alerts when new articles cite this article - sign up in the box at the } \\ & \text { Service }\end{aligned}$ top right corner of the article or click here. 